49. Dollinger: Suspension und Stützpunkte künstlicher Glieder. 42. Chir. Kongr. 1913.

46-48. Die Arbeiten eignen sich weder zu kurzem Referat, noch gebören sie ins orthopädiscbe Gebiet. Sie seien nur der Vollständigkeit wegen angeführt.

44. Die Prothese soll die Fortsetzung des Stumpfes sein, deshalb muss sie detuselben gut anmodelliert werden. Um dies besser zu erreichen, modelliert Dollinger an das Gipsmodell des Stumpfes bestimmte Stütz- und Druckpunkte.

\title{
An hang.
}

Bücher über Verbände und portative Apparate, die in Bd. XII. dieses Archivs bereits referiert sind.

50. Lewy: Die ärztliche Gipstechnik. Seite 193.

51. Steinmann: Die Nagelextension rer Knochenbrüche. Seite 195.

52. Riedel: Eine eivfache Extensionsmethode zur Behandlung der Beinbrüche im Geh-Verband. Seite 195.

\section{Bücher.}

Treves-Keith, Chirurgische Anatomie. Nach der sechsten englischen Ausgabe überaetzt von Dr. A. M ülberger. Mit 152 Textabbildungen. $478 \mathrm{~S}$. Verlag von Julius Springer, Berlin 1914. Preis gebunden M. 12.-.

Wenn auch zugegeben werden muss, dass selbst die geringfügigste wissenschaftliche Einzelheit einmal unerwartet Bedeutung gewinnen kann, so gibt es doch gerade in der Anatomie eine grosse Menge von Tatsachen, welche der Mediziner leicht, gern und meistens auch ungestraft vergisst. Was das Studium der systematischen Auatomie als der Grundlage aller Heilkunst so trocken macht, ist ja der Umstand, dass der Lernende nicht absehen kann, was er später brauchen wird, was als toter Ballast über Bord geworfen werden kann. Wenn die topographische oder die chirurgische Anatomie sich grösserer Beliebtheit erfreuen, so liegt dies daran, dass hier die Brücke von der Wissenschaft zum Leben, vom blossen Erkennen zum Verstehen und äratlichen Handeln gsschlagen wird. Wir haben in Deutschland keinen Mangel an guten Werken über chirurgisch-topographische Anatomie. Dennoch dürfte das engrlische Buch bei uns Eroberungen machen, und zwar gerade wegen der Eigenschaften, welche es von unseren deutschen Werken unterscheidet. Der Engländer ist eben, wie Payr in der Vorrede hervorbebt, auch in seiner Wisseuschaft der geborene Praktiker. Er lerut uicht wie wir Anatomie, um diese später auf das ihm zunächst noch fremde Gebiet der Praxis zu übertragen, sondern aus den Notwendigkeiten der äratlichen Praxis heraus kommt er zum geistigen Erfassen der theoretischen Probleme. Das vorliegende Buch wurde geschrieben, um vor allen dem Studierenden eine Vorstellung von dem verhältnismässigen Wert les anatomischen Tatsachenmateriales zu geben, dann aher auch, um die avatomischen Kenntnisse des in der Berufsarbeit stebenden Arztes aufzufrischen und neu zu beleben. Bei dieser Betonung praktischer Ziele muss die englische Besonderbeit als ein eurschiedener Vorzug des Werkes angesprochen werden. Unbeschaiet deutscher Gründlichkeit wird auch bei uns die Art des englischen Autors, das Wesentliche zu unterstreicben, das Unwesentliche fortzulassen und das Ganze knapp auf die Bedürfnisse des ärztlichen Handelus zuzuschneiden, lebbaften Anklang fincien, und es dürfte nichts schaden, wenn es auch etwas Schule machen würde. Dass die Beschränkung des Stoffes nicht zu 
oberfächlichem Halbwissen verleite, dafür bürgt der ausgezeichnete wissenschaftliche Geist des Verf., welcher die Erscheinungen mit Sinn und Interesse umgibt. In der Tat bietet die Lektüre des Buches neben reicher Belehrung auch einen ausgewählten Genuss. Durch ganz vorzügliche Abbildungen wird die Anschaulichkeit der Darstellung gefördert. Auch der trefflichen Cibersetzung von Dr. A. Mülberger sowie den Illustrationen von Dr. Kleinsehmidt und Dr. Hörhanmer aus der Payr'seben Klinik ist ein gutes Teil am Gelingen des verdienstvollen Werkes zu danken.

$\mathrm{K}$ ünne (Steglitz).

Gocht, Handbuch der Röntgen-Lehre, IV. umgearbeitete und vermehrte Auflage. Stuttgart 1914, bei Ferd. Enke. M. 13.80 .

Drei Jahre nach der letzten Auflage wurde eine neue nötig: bei dem Überfluss an Röntgen-Lehrbüchern wabrlich ein gutes Zeichen! Die neue Auflage ist den neuesten Fortschritten entsprechend ramentlich in den Abschnitten über Rïntgeninstrumentariuı und Röntgenröhre erweitert worden; auch die Kapitel über Anatonie und Physiologie, Geburtshilfe und Gynäkologie, Untersuchung des Magen-Darmtraktus, endlich die Röntgentherapie, besouders die Tiefentherapie haben eine besondere Ausgestaltung erfahren.

Text und Abbildungen mussten trotz aller Kürze und Knappheit etwas vermebrt werden, um die Höhe moderner Wissenschaft zu erreichen.

$$
\text { W. Becker (Bremen). }
$$

Wetterer, Handbuch der Röntgentherapie. Zweite umgearbeitete und erweiterte Auflage. 2 Bände. Leipzig 1914, bei Otto Nemnich. Preis in Leinenband Bd. 1 M. 18,一, Bd, 2 M. 26,一.

Von dem grossen Handbuch Wetterers, dessen erste Auflage bereits in Bd. VII dieses Archivs eingehend gewürdigt wurde, liegt jetzt eine neue Auflage vor. Ihre völlig neue Durcharbeitung und wesentliche Vermehrung kommt äusserlich dadurch zum Ausdruck, dass sie in 2 getrennten Bänden erschienen ist, von denen der erste den allgemeinen, der zweite den speziellen Teil der Röntgen. therapie enthält.

Neu bearbeitet sind vor allem 3 verschiedene Abschnitte der früheren Auflage: die Röntgentechnik, die Tiefenbestrahlung und die Radiumtherapie. Der tecbnische Teil enthält die neuesten Forschungen über das Wesen der rätselbaften Strahlen und gibt gleichzeitiy eine Übersicht über alle Verbesserungen und Neukonstruktionen, die in den letzten 5 Jahren erschienen sind.

Die Tiefenbestrablung steckte damals noch in den Wirideln. Daher galt es hier eine reiche Arbeit zu bewältigen, um im allgemeinen und speziellen Teil den verschiedenen Anforderungen, die das neue Verfahren an Technik, Praxis und klinische Erfahrung stellt, gerecht zu werden.

Daselbe gilt von der Radiumtherapie; auch hier ist im Laufe der letzten Jahre so viel geschrieben worden, dass es keine geringe Mühe bedeutet, die verschiedenen Forschungen bei der Neubearbeitung zusammenfassend darzustellen. Denn soviel steht längst fest: mag auch die Rndium- und Mesothoriumbestrahlung nur einen kleinen Teil all der Hoffnungen, die man im vorigen Jahre davon erwartete, erfüllt haben, als Ergänzung der Röntgentherapie ist sie bereits heute wichtig und unentbehrlich.

Im übrigen gilt von der neuen Auflage das bereits früher Gesagte: der Fleiss und die Gründlichkeit, mit der das grosse Material bewältigt wurde, verdienen dieselbe Anerkennung wie die äussere Ausstattung. War die erste Auflage schon reichlich mit Abbildungen versehen worden, so ist bei der zweiten die Zahl der Textfiguren und der Tafeln in Mehrfarbendruck und in Schwarzdruck mehr als verdoppelt worden! Auch sei auf das ausführliche Literaturverzeicbnis am Schluase hingewieset, das nicht weniger als 138 Seiten beansprucht. Wir wünschen der neuen Auflage einen zahlreichen Leserkreis.

W. Becker (Bremen). 
Universität Zürich, Einweihung 1914. Festgabe der Medizinischen Fakultät. Zürich 1914. Schulthess \& Co. 286 Seiten, mit Abbilduugen. Ungeb. 4,-. Die Festscbrift enthalt folgende Arbeiten:

Eichlorst, Infektionskrankheiten und Volkswohlfahrt.

Der Aufsatz behandelt nach kurzen allgeneinen Ausfübrungen die diesbezüglichen Verbältnisse der Stadt Zürich. Die Pest ist seit langen Zeiten unbekannt, die letzte Cholersepidemie war 1867, der letzte vereinzelte Fall 1892. Die Erkrankungen an Pocken haben sich seit Abschaffung des Impfzwanges im Jahre 1883 merkwürdigerweise nicht wesentlich vermehrt, was damit erklürt wird, dass ein sehr grosser Teil der Bevölkerung sich freiwillig impfen lässt. Seit die städtische Wasserleitung gründlich verbessert ist, kommt Typhus fast nur bei $\mathrm{Zu}$ gereisten vor. Nackenstarre und Kinderlähmung blieben selten, Flecktyphus oder Febris recurrens fehlten gänzlich. Zürich ist arm an Diphtherie, die Scharlachund Mremerkrankungen schwanken.

Gaule, Der Wärmebaushalt.

Die tieriscbe Wärme in ihrer Bedeutung für das Lebensproblem und die individuellen Lebenaäusserungen, die Einhaltung des Wärmeoptimums durch das regulierende Wärmezentrum, die reaktive oder künstliche Steigerung der Temperatur als Kampfmittel gegen eindringende Schïdlichkeiten.

Haab, Das Brillentragen.

Bekämpft alte Vorurteile. Ein richtiges Augenglas ist nicht schädlich, sondern schont und erhält den guten Zustand des Auges, es ergänzt physiologische oder pathologische Mängel unseres Sehapparates.

Wyder, Der Geburtenrückgang.

Erschwerung des Daseinskampfes und steirgende Genusssucht aind die Ursachen der gewollten, Geschlechtskrankheiten die der unfreiwilligen Unfruchtharkeit.

Ruge, Vom Bau des menscblichen Körpers.

Betrachtungen über dio durch den aufrechten Gang bewirkten Umformungen der einzelnen Organe.

Bleuler, Die A mbivalenz.

Cloette, Über Angewöhnung an Medikamente und Gifte.

Die Giftimmunität kaun eine angeborene sein, eine durch - Krankheit bedingte oder eine systematisch erworbene. Die der Gewöhnung zugrunde liegenden Vorgänge sind bei den einzelnen Stoffen zum Teil ganz verschiedenartige.

Silbersehmidt, In fek tionskrankheiten und Disposition.

Verf. bezeichnet als eine der wichtigsten Aufgaben der Zukunft neben der Erforschung der Krankheitserreger die wissenschaftliche Ergründung der Disposition. Die individuelle Disposition beträgt bei Masern $90 \%$, bei der epidemischeu Genickstarre ist sie sehr gering. Wie Mischinfektionen die Disposition erhöhen, könnea sie diese auch, z. B. beim Milzbrand, herabsetzen.

Sauerbrueh, Die Behandlung chirurgiseher Erkrankungen mit künstlicher Bestrahlung.

Verf, waint vor der Üherschälzung der natürlichen oder künstlichen Strablenbehandlung. Die Heliotherapie leiste Beachtenswertes, doch sei sie kostspielig und langwierig. Heruntergekommene Tuherkulöse finden auch häufig bei einfacher Krankenhausbehandluug Besserung und Heilung. Das Wesentliche sei wohl die gründliche Änderung des Stoffwechsels. Ebensowenig wie das natürliche Sonnenlicht seien Röntgen- und Radiumstrahlen bisher als sicher wirkende Heilmittel zu 
betracbten. Bei bösartigen Neubildungen gebühre nocb imner dem Messer der unbedingte Vorrang. Jedem neuen Heilmittel gegenüber sei objektive Prüfung am Platze. Der Kliniker, welcher voreilig Hoffnungen erweckt, die zu Enträuschungen führen, schädigt das Ansehen des ärztlichen Standes.

Busse, Krebsforschung und Krebsbekämpfung.

Zangger, Über allgemein notwendige Kenntnisse und zu wenig bekante Ursachen der Kohlenoxydvergiftung.

Der Aufsatz gipfelt in der Forderung staatlicher Bekämpfung der zahlreichen Kohlonoxydgefahren.

Feor, Säuglingssterblichkeit und Geburtenrückgang.

v. Monakow, Betracbtungen über enger begrenzte "Sprachzentren“ im Cortex.

Felix, Die Missbildungen der Müller'schen Gänge und ihrer Ab$\mathrm{köm}$ mlinge.

Miiller, Die Arceriosklerose und ihre Behandlung.

Sommer, Balneologiscbe Skizzen.

Schulthess, Die Anstalt Balgrist.

Die Anstalt besteht aus dem sogenannten Haupthause und der Poliklinik. Sie ist bestimmt „für Kinler, in beschränkler Anzahl auch für Erwachsene, welche mit Leiden behaftet sind, die orthopädiscb behandelt werden könven". Sie verfügt demgemäss über Einrichtungen zur ärztlichen Behandlung, zur Erziehung und Schulung, sowie zur Vorbereitung für einen Beruf. Die Anstalt hat Platz für 85 Kranke, in der Poliklinik werden täglich 80-90 Personen behandelt. Wobltätig berübrt die Vermeidung des Wortes „Krüppelheim“. Kü nne (Steglitz).

Ludwig Jehle, Die Albuminurie. Klinische und experimentelle Beiträge zur Frage der orthostatiseb-lardotischen und der nephritischen Albuminurie. Mit 35 Abbildungen in Text und 2 Abb. auf einer Tafel. Berlin, Julius Springer, 1914. Preis M. 4,-

"Wir müssen dem Autor für seine hochinteressante Arbeit dankbar sein und wünschen, dass sie zum Nutzen der erkrankten Menschheit noch zu weiteren diesbezüglichen Arbeiten Anlass geben werde."

(Aus der Besprechung der Arbeit Jehles von Scholder und Weith, siehe dieses Heft S. 33t).

\section{Journalliteratur.}

Rudolf Selig, Vorschlagzurextraperitonealeu Resektiondes Nervus obturatorius bei Spasmen der Adduktoren. Zeitschr. f. angewandte Auatomie und Konstitutionslehre. Bd. 1, Heft 2, 1913.

Verf. hat an 30 Leichen die extraperitoneale Resektion des Nervus obturatorius ausgeführt. Das Verfahren wird als technisch leicht bezeichnet. Bei doppelseitiger Affektion gestaltet sich der Eingriff folgendermassen: hoher Blasenschnitr, Zurückschieben des Bauchfells, stumpfes Vordringen nach der seitlichen Beckenwand. Der ziemlich dicke Nerv wird dann am unteren Rande des Schambeins gefunden, von der Arteria und Vena obturatoria isoliert und durchscbnitten.

Künne (Steglitz).

Rudolf Selig, Heidelberg, Die intrapelvine extraperitoneale Resek. tion des Nervus obturatorius und anatomische Studien über 
die Topographie dieses Nierven. Archiv für klin. Chirurgie. Bd. 103, Heft 4.

Verf, kommt bei einer kritischen Betrachtung der bisher üblichen Behandlungsmethoden zu einer absoluten Verwerfung der subkutanen Tenomyotomie der Adduktoren. Nach Leichenversuchen stellt diese Operation sich dar als eine rohe, völlig unkontrollierbare Durchschneidung von Muskeln und Sehnen säntlicher Adduktoren, des Nervus obturatorius bzw. seiner Äste und fast aller zur Fruährung der Adduktoren nötigen Blutgefässe. An Stelle des Spasmus tritt somit die ischamische Kontraktur. Misserfolge und Rezidive nach Sebnendurchschneidung sind denn auch nehrfach berichtet worlen. Stoffel selbst sab nach seiner Obturatoriusresektion niemals Rezidive, und er erklärt die von anderer Seite beobachteteu damit, dasz wabrscheinlich der Ranus profundus oder accessorius überseben wurde, oder dase sonst eine Anomalie vorlag. Jedenfalls hat auch die Stoffel'sche Operation den Nachteil, dase sie ein grosses Wundbett schafft und zu regressiven Veränderungen Veranlassung gibt. Selig schlägt deshalb vor, den ungeteilten Obturatoriusstamm durch Bauchschnitt am Rande des Rectus im Becken aufzu. suchen. Die Fascia transversa und das Peritoneum werden seitlich weggeschoben. Der Nerv verläuft an der lateralen Seite des Beckens, ganz wenig unterhalb der Linea innominata. Als Vorzüge seines Verfahrens gibt der Verf. an: die leichte Auffindharkeit des Nerven, die Möglichkeit der Dosierung sowie die Schonung der Gefäspe, die weite Entfernung des Operationsschnittes von riem spastischen Gebiete, das Uuversebrthleiben der Adduktorenmuskulatur, die rasche Wundheilung und die damit verbundene schnelle Herstellung der Gehtähigkeit.

Künne (Steglitz).

Hans Virchow, Der Zustand der Rückenmuskulatur bei Skoliose und Kyphoskoliose. Zeitschr. f. orthop. Chirurgie, Bd. 34, 1914.

Die ausserordentlich gründliche Untersuchung wurde an zwei rein skoliotischen und einem kyphoskoliotischen Rücken, den gleichen, dereu Runpfskelette Verf. nach Form zusammengesetzt und beschrieben hat, vorgenonmen. Drei gute Abbildungen verauschaulichen den reichhaltigen urd ungemein lehrreichen Text. Allerdings sind die Ergebnisse, zu denen Verf. durch seine mübselige Arbeit geführt wird, derarh, dass der Orthopäde sie weder in positiven noch im negativen Siune, weder für seine Anschauung noch für sein Handeln irgendwie unmittelbar ausbeucen könnte. Bei schwerer Skoliose zeirren sich die oberflïchlichen Rückennuskeln wenig verschieden von norinalen, an den tiefen Rückennuskeln wird eine gewisse Dürftigkeit konstatiert, die Degeneration steht aher in gar keinem Verbältnis zu den Grade der Deformität. Hatte doch bei vorliegeuder Untersuchung die schwerste Skoliose die gesundesten Rückenmuskeln! Degeneration kam sowohl an verkürzten wie an unverkürzten Muskelpartien ohne erkennbare Beziehungen vor, und zwar gibt sich die Entartung als bindegewebige oder als fettige zu erkennen. Virchow schliesst mit Bestimmtheit, dass die Muskelveränderungen sekundär durch den Zustand des Skeletts herrorgerufen sind. Allerdings soll auch diese sekundäre Insuffizienz der Muskulatur ibrerseits wieder die Skelettdeformität steigern. Nirgends zeigt sich die Spur einer nüłzlichen und zweckmässigen Anpassung. Wir lernen aus Virchows Ausführungen, dass die Muskel- und Knochenverhältnisse bei der Skoliose noch viel komplizierter sind als wir uns vorzustellen plegen, und dass wir im besonderen vou der korrektiven Wirkung gymnastischer Methoden nicht viel erwarten dürfen. K ünne (Steglitz).

W. Lovett, The bistory of scoliosis. American journal of orthopedic surgery. Juli 1913.

Die Geschichte der Skoliose reicht bis zu den Anfängen der Medizin zurück. Hippocrates gab der Krankeit den Namen, batte jedoch ron ibrem Wesen 
nur sehr unzureichende Vorstellungen. Die endgültige Abgreuzung der seitlichen Verbiegungen von den spondylitischen Kyphosen wurde erst durch $\mathrm{P}$ ott's $\mathrm{Be}$ schreibung der letzteren im Jahre 1779 bewirkt. Eine wirkliche Skoliosenbehandlung durch Redressement existiert erst seit 1875. Die Anfänge der gymnastischen Behandlung sind schon bei Hippocrates zu finden. Künne (Steglitz).

Lovett, The treatmeut of scoliosis. Boston medical and surginal journal, Bd. 169, Nr. 18, 30. Oktober 1913.

Whas wir Skoliose nennen, umfasst zwei ganz verschiedeue Zustandsänderungen, welche in Interesse wissenschaftlicher Verständigung, Prognosestellung und Behandlung streng auseinandergehalten werden solltev. Wir müssen grundsätzlich unterscheiden zwischen der funktionellen und der strukturellen Skoliose. Bei der funktionellen Skoliose, auch Haltungsskoliose, schlechte Haltung, totale Skoliose oder wie Verí. vorschlägt, aucb falsche Skoliose genaunt, besitzt der Patient eive normal gebaute Wirbelsäule, das Röntgenbild zeigt keine Veränderungen der einzelnen Bausteine, die Seitenausbiegung verschwindet beim Iiegren. Ganz anders bei der strukturellen oder organischen Skoliose, welche der Verf. im Gegensatz zur ersteren auch als wahre Skoliose bezeichnet wissen will. Hier ist die Wirbelsaiule in ihren Gliederungen primär veründert, das Röntgenbild zeigt diese Abweichungen, und die Kurve verschwindet nicht beim Niederlegen des Patienten. Die diagnostischen Merkzeichen der falschen Skoliose sind also 1. eine einzige Kurve, meist linkskouvex, 2. Höhersteben der konvexseitigen Schulter, Rückwärtsdrehung der bonvexseitigen Schulter gegen das Becken, geringes Höhersteben der konkaven Seite bei Vorwärtsbeugung (sogenannte Konknvtorsion). Die wahre Skoliose wird daran erkannt, dass die Wirbelkörper nach der konvexen Seite der Krümmung gedreht sind, und dass bei Vorwärtsbeugung die der konvexen Krürmung entsprechende binter Thoraxpartie stark hervorspringt. Die falsche Skoliose ist ein Maskelproblem, sie wird daher mit Gynnastik behandelt und geheilt. Die wahre Skoliose ist aber kein Muskelproblem! Folglich ist Gym. nastik nicht nur unnütz, sondern möglicherweise scbëllich. Frstere kommt zustande unter dem Einfluss feblerhaften Stehens und Sirzens, geringfügiger anatomischer Unregelnässigkeitev an Beinen, Becken, Kopf, Wirbelsäule, durch ungleiche Ausbildung des Gesichts- oder Gebörssinnes, durch Beschäftigungshaltungen. Letztere zählt unter ihre Ursachen: angeborene Missbildungen der Wirbelsäule oder ihrer Anbangsgebilde, Empyem, Kinderlähnung, Rachitis, In gewisen Fällen sch verer struktureller Skoliose unss man eine vermiuderte Widerstandsfähigkeit der Knochen gegen Deformierungen aunehmen. Ein zu kurzes Bein kann bei normalem Knochensystem nicht die Ursache einer schweren Skoliose sein, wohl aber kann eine solche Beinverkürzung bei vorhandener abnormer Knochenschwäche eine wahre Skoliose höheren Grades hervorrufen.

Die einzig rationelle Therapie der wabren Skoliose ist das Redressement. Dass Nuskelkontraktionen deforme Wirbelsäulen aufrichten oder borrigiert erhalten, kann bei der Skoliose ebensowenig angenommen werden, wie wir solche Wirkungen beim Klumpfuss oder beim Bein erwarten. Verf. bespricht sodann die einzelnen Methoden des Redressements unter vertikaler und borizontaler Suspension, bei maximaler Extension, die überkrümmenden Verfahren bei Kyphose der Wirbelsäule in Bauch-, Rücken- und Seitenlage sowie die Methode der Drehung nach Forbes, über die sämtlich das Urteil noch nicht abgeschlossen jst. Immerhin geben alle diese Methoden so schöne Erfolge, dass schon heute die Anschauung ausgegprochen werden kann, die Zukunft der Skoliosenbehandlung beruht auf der Weiterent. wickelung des forcierten Redressements. Natürlich wird die Nachbehanilung mit Massuge und Gymnastik nie ihre hohe Bedeutung verlieren.

K ün n e (Steglitz). 
Schanz, Ein Besuch bei Rollier. Zeitschr. f. orthop. Chirurgie, Bil. 34, 19 i4.

Ein persönlicher Besuch bei Rollier hat $\mathrm{Schanz}$ zu einem begeisterten Anbäuger und Schilderer des dort geübten Heilverfahrens gemacht. Er gibt gern zu, dass zeine schon nicht geringen Erwartungen von dem Gesebenen weit übertroffeu wurden. Nicht genug weiss er den prächtigen Allgemeinzustand der Patienten, die sammetartige Haut, die elastische Muskulatur, die gute Entwickelung des Brustkorbes, den frischen Glanz der Augen und das blanke Weiss der Zähne zu rühmen. Interessant ist auch, dass mit den primitivsten orthopädischen Mitteln, mit Kissen, einfachen Zügen und Matten hier die wunderbarsten Wirkungen auf Deformitäten ausgeübt werden. Die Sonnenkraft erbält selbst schwer zerstörten Gelenken die Beweglichkeit, lägst seit Jabren totkranke Glieder voll und rundlich erscheinen. Aber nicht nur der Sonne sind diese Erfolge zuzuschreiben, sondern in erster Linie - so meint Schanz - Rollier's Regime. Hier kommen die mit grosser Konsequenz eingehaltene Ruhelage, die vorzugэweise vegetarische Diät, die Iröhenlage, schliesslich Rollier's ganze ärztiche Persönlichkeit als mächlig unterstützende Faktoren in Betracht. Wenn wir Rollier's klinischen Grundsätzen nacheifern, so lässt sich auch in unseren weniger freundlicben Himmelsstrichen ohne die Sonne Leysins Erfreuliches erreichen. Künue (Steglit\%).

Gaugele und Schïssler, Unsere Erfahrungen mit dem Friedmann. schen Tuberkulosemittel. Deutsche med. Wocheoschr. 1914. Nr. 17.

An 34 Kindern, die hauptsächlich an Knochentuberkulose litten, wurden ausgeführt: 6 intramuskuläre Seruminjektionen (Nr. 1 grün), 26 Simultaninjektionen (Nr. II blau intravenös + Nr. III gelb intranuskulär), 2 Injebtionen mit Serum III. Ein Kind starb an Miliartuberkulose, der Exitus schien hier, wenn nicht direkt verureacht, so doch beschleunigt durch die Injektion. Die Allgemeinerscheinungen waren unmittelbar nach der Injektion vielfach schwerster Art. In 21 Fällen trat starke Abszessbildung auf, die auf die Uureinbeit des Präparates zurückgeführt wird. Nach Abschluss der Eiterung wurde allerdings eine Besserung des Aligemeinbefindens wahrgenommen. Die Pirquel'sche Probe blieb bei allen Palienten positiv, die Röntgenbilder ergaben keine Besserungen der Knochen- und Gelenkverhälınisse.

K ü a ne (Steglitz).

Iovett und Fish, The outdoor treatment of surgical tuberculosis. Boston medical and surgical journal, Bd. 169, S. 145-151, 31. Juli 1913.

Die Freiluftbehandlung der chirurgischen Tuberkulose ist, von der Heliotherapie abgesehen, noch nicht in ihrer eigentümlichen Bedeutung voll gewürdigt worden. Im Massachusetts-Hospital für verkrüppelte Kinder verbringen die Insassen 'Tag und Nacht, Sommer und Winter, auch die Zeit des Unterrichta in Freien, bezw. in Räumen, welche durch ibre Bauart und lüftungsvorrichtungen alle Vorteile der freien Iuft darbieten. Die Wirkungen auf Allgemeinbefinden, Ernährungszustand, Blutbescbaffenheit und örtliche Erkrankung sinł ganz vorzügliche. Erkältungskrankheiten sowie Infektionen sind seltener als bei anderen Schulkindern. Mau hat die Erfahrung gemacht, dass man Kinder unter 3 Jahren und Patienten nach Operationen nicht niederen Temperaturon aussetzen darf. Die Verff. betonen den hoben Wert des dauernden Freiluftlebens auch für die gesunde Schuljugend.

Künne (Steglitz).

Lovett, Principles of the treatment of infautile paralysis. The journal of the American medical association, 24. Jan. 1914, Vol. 62, pp. 251 bis 254 .

Der Nutzen des Hexamethylentetraming bei frischer Poliomyelitis ist noch nicht sicher erwiesen, dennoch kann es für verdächtige Fülle empfohlen werden. Leicbtere Fälle bedürfen zwei bis drei Wochen der Rube, scbwerere so lange, bis alle 
Empfindlichkeit verschwunden ist. Schwerere Attacken sind meist von schwereren Lähmungen gefolgt und umgekehrt, doch ist diese Regel nicht ohne Ausnahmen. Der Ausgang der Krankheit ist daher selbst bei frühzeitiger sachgemässer Behandlung nicht vorber zu bestimmen. Das Rekonvaleszenzstadium teilt Lovet aus praktischen Gründen in die noch emptindliche Phase und in die Phase nach Verschwinden der Enpfindlichkeit. Für die erste Phase ist nichts weiter nötig als Ruhe, wenn möglich im Freien, Lagewechsel, vorsichtiges Aufsetzen, warme Bäder, Verhütung der Spitzfusskontraktur. Erst in der zweiten Phase, die nach zwei bis drei Monaten gewöbnlich beginnt, treten Massage, Elektrizität und Übungsbebandlung in ihre Rechte. Vor Ablauf von 4 Wochen sollte die pbysikalische Behandlung jedeufalls nicht angewandt werden. So früh wie möglich ist der Patient sodann durch Apparate gehfähig zu machen. Gerade der frühe Gebrauch der Apparate schützt den Kranken ain besten vor späterer dauernder Abhängjgkeit von mechanischen Hilfsmitteln. Muskelatrophie ist nicht zu fürchten, denn die Fortbewegung, selbst in Schienenapparat, ist die beste Muskelübung. Für die operative Behandlung fordert Lovet t, dass zwei bis drei Jahre seit dem Anfall vergangen seien, eine Forderung, von der Ref.' glaubt, dass sie sich auch bei uns durchsetzen wird, deun die endliche Wiedererlangung der Funktion geschieht auf Grund von dreierlei verschiedenen Vorgängen, nämlich durch Wiederwegsamwerden der erkrankten Nervensubstanz, durch Benutzung benachbarter Nervenfazerbahnen, durch die kompensierende Tätigkeit gesund gebliebener Muskeln. Der Fuszarthrodese zieht Lovett die partielle Fixierung durch künstliche Seidenligamente vor, da schwere sekundäre Deformieruugen zuweilen die Folgen der Versteifung sind. Wenn Lovett die Kuiearthrodese wegen der zu fürchtenden Wachstumsstörung ablehnt, so wird das Vorliegen dieser Gefahr durch die zahlreichen Erfahrungen der Biesulski'sehen Klinik nicht bestïtigt. Andererseits sprechen sicher praktische, ästhetische und psychische Gründe gegen die künetliche Versteifung des Kniegelenks.

Künne (Steglitz).

Schanz, Zur Ätiologie und Therapie der Arthritis deformans. Zeitschr. f. orthop. Chirurgie, Bd. 34, 1914.

Schanz ist der Überzeugung, dass die Arthritis deformans eine Verbrauchskrankheit darstelle. Dabei versteht er unter Verbrauchskrankheit eine solche, welche ohne abnorme Einwirkung lediglich durch den normalen Gebrauch früher oder später bei jeden Menschen zur Ausbildung kommt. So erkläre es jch, dass die Arthritis deformans bei Menschen, die viel auf den Beinen seien, so häufig vorkomme. Bei der Arthritis der Tabiker wird die schnellere Abnutzung aus den Sensibilitātsstörungen und dem stampfenden Gange verständlich. Die entzündlichen Erscheinungen sind Reaktionserscheinungen, die Wucherungen Heilbestrebungen des Organismus. Abnorme Gelenkstellungen, ferner Gelenkflächen inkongruenzen bedingen, schief eingestellten Maschinenteilen vergleichbar, ebenfalls schnelleren Verbrauch und damic Artbritis. Die Therapie hat demnach die Aufgabe, den übermässigen Verbrauch einzuscbränken, in erster Linie geschieht dies durch Entlastung. Die Stoffivechselvorzüge sind durch Priessnitzumschläge, sanfte Massage und Elektrisation anzuregen, dagegen soll jede Reizung des Gelenkes, etwa durch direkte Massage oder Heisshuftbehandlung vermieden werden. -

Gaugele, Über die sogenannte Entbindungslähmung des Armes. Zeitschr. f. orthop. Chir., Bd. 34, 1914.

Verf. bringt 4 Fälle mit Röntgenbildern. In allen 4 Fällen wurden Epiphysenverleczungen konstatiert. Gaugele schliesst sich der Anschauung Lange's an, dass bei dem Krankheitsbilt der Entbindungslähmung meist keine echte Lähmung vorliege, sondern dass es sich hauptsäcblich um Verletzungen der Kapsel 
handele, die später dann zu Scbrumpfung und Kontrakturbildung fübren können. Epiphysenverletzungen kommen meist vor, doch sind sie ebenso wie etwaige Knochenverletzungen nur als komplizierende Begleiterscheinungen anzusehen. Die Prognose ist in frischen Fällen gur, in alten besser als früher. Für die ersteren sind Abduktionastellung von $90^{\circ}$ bei starker Auswärtsdrehung, später Maszage und passive Ubungen zu empfehlen; in veralteten Fällen kommen Redressement, Durchschneidung des Pectoralis major und Osteotomie des Humerus in Betracht.

Künne (Steglitz).

Gaugele, Uber die Behandlung der angeborenen Hüftgelenksverrenkung. Zeitgchr. f. orthop. Chirurgie, Bd. 34, 1914.

Die Rückkehr des Gliedes aus der Repositionsstellung in die Normalstellung darf nicht dem Patienten überiassen bleiben, sondern stellt den wichtigsten Teil der Nachbehandlung dar. Durch die genaue Befolgung dieses Grundsatzes, der schon von Schanz und Hoeftmann vertreten ivurde, hat Verf. es auf eine Heilungszifter von $100^{\circ} \%$ gebracht. Er verwendet nach drei Gipsverbänden von je j Wochen Dauer eine Luxationsbandage, welche im wesentlichen aus einem Beckenkorb und darin befindlicher, verstelibarer und feststellbarer Oberschenkelschiene besteht. Der erste Verbund wird in Lorenz'scher Primärstellung von $90^{\circ}$ Abduktion, $90^{\circ}$ Flexion, $0^{\circ}$ Rotation, der zweite in leichter Eiuwärtsrotation und geringer Verninderung der Abduktion, der dritte je nach dem Röntgenbefunde in vermehrter oder verminderter Abduktion angelegt, Die Nachbehaudlung mit der Luxationshandage dauert $10-15$ Wochen. Die Einrenkung hat stets so schonend wie möglich zu geschehen; in diesem Falle scheinen schwerere Kopf- und Halsdeformitäten nicbt zur Entwickelung zu kommen. Der Antetorsion misat G a u gele keine so hohe Bedeutung für den Enderfolg bei. Künne (Steglitz).

Wegner, Über die Luxation des Fusses im Talokruralgelenk nach o be n. Beiträge zur klinischen Chirurgie, Bd. 88, Heft 2, 1913.

Den wenigen bisher beschriebenen Fällen fügt Verf. einen Fall hinzu, welcher als erster auch röntgenologischer Betrachtung unterzogen wurde. Der kräftige und sonst gesunde Mann fiel seitlich um, während sein rechter Fuss zwischen die Sprossen einer Leiter gequetscht wurde. Die Symptome bestanden in kolossaler Verbreiterung des Fussgelenks, starkem Vorspringen des medialen Knöchels, Auswärtrotation und Abduktion des Fusses. Die Reposition gelang leicht durch Zug nach unten, die Heilung war eive vollstäudige. Das Röntgenbild zeigt das Sprungbein zwischen Tibis um Fibula eingekeilt, jedoch ist nur die Tibia, von deren unterem Ende ein Stück abgesprengt ist, aus ihrer Lage verschoben, während die Fibula an ihrer Stelle geblieben ist. Die Prognose der seltenen Verletzung wird als günstig bezeicbnet. K ünne (Steglitz).

Iuskat, Berlin, Fusserkrankungen bei Schulkindern. Zeitschr. f. Schulgesundheitepflege, 1913, 26. Jabrgang, Nr. 10.

Von den zahlreichen Missbildungen, den tuberkulösen und den Lähmungserkrankungen des Fusses beansprucht das Hauptinteresse wegen seiner Häufigkeit der Plattfuss. Er kommt vor angeboren in $4,3 \%$, paralytisch in $3,1 \%$, traumatisch in $4,9 \%$, rachitisch in $3,1 \%$, statisch in $88,9 \%$ der Fälle. Die Anschauung vom physiologischen Plattfuss aller Neugeborenen gilt als widerlegt, ebenso wie die von Plattfuss als Rassemerkmal. Ewald fand in israelitischen Schulen nicht mehr Plattfüsse als in anderen. Der Prozentsatz der plattfüssigen Schulkinder ist ein ausserordentlich hoher, nach verschiedenen Autoren zwischen 10 und $37^{\circ}: 0$. Die Zahl nimmt im allgemeinen nach den oberen Klassen zu. Da der Plattfuss Militär- und Berufstauglichkeit in bohem Masse einschränkt, sind weitgebendste prophylaktische Massnahmen, die vor allem in das Aufgaben- 
gebiet des Schularztes fallen, geboten. Überanstrengungen durch Stehen und Gehen, sowie bei gewissen Turnubungen sind zu vermeiden, das Schuhwerk soll am Innenrande am längsten, die Sohle ev. etwas erböbr sein, Fusspflege durch Bänder, geeignete Muskelübungen ist zu empfehlen; in schweren Fällen hat spezialärztliche Behandlung rechtzeitig einzusetzen.

Künne (Steglitz).

Kraft, Wetterer und Wiesner, Archiv für physikalische Medizin und medizivische Technik. FIII. Bu., Heft 4, Leipzig 1914 bei Otto Nemnieh, Eiuzetpreis 3.50.

Originalien:

1. Schmidt, Zur Strahlenbehandlung des Krebses.

Es ist bisher noch kein Beweis dafür erbracht worden, dass Radium oder Mesothorium wirksamer seien als Röntgenstrahlen. In graden Gegenteil sah Bumm die besten Erfolge bei Fällen, die nur mit Rüntgenstrahlen behandelt worden waren.

2. Knox, Die Radiumtherapie bei malignen Tumoren.

In den Anfangsstadien von Krebs ist unbedingt der operativen Methode der Vorzug zu geben; Radium komm als bestes Hilfsmittel bei allen Fällen in Betracbt, sowobl als Prophylaxe nach Operationen, als auch, wenn keine Operation ausgeführt war. In inoperabled Fällen kann es unter Umständen zur Operabilität führen. Es ist za beachten, dass Röntgenstrahlen in mauchen Fällen genau dasselbe leisten wie Radium.

3. Dessauer und Wetterer, Bemerkungen zur Anwendung der barten Röntgenstrablen.

Für die Hypertrichosis sewohl als für die Erkrankungen der Haare sind die harten Strahlen die geeignetsten, während für Ekzeme, Psoriasis, Prurigo, Pruritus, Lichen ruher etc. nur mittelweiche Strahlen in Betracht kommen.

4. Schnép, Die Entwickelung deselektrischen Entfettungsverfahrens und mein Degrassator.

Beschreibung des von Verf. konstruierten Apparates mit historischem Rückblick auf die elektrische Entfeltungstherapie.

Zeit- und Streitfragen; Kritik; Referate.

$$
\text { W. Becker (Bremen). }
$$

Coakley, Byron, 1. Li fe without organs. Cuakley's Archives, Vol. 1, April 1914, Nr. 1.

Die Versuche des Autors an Fröschen, Eidechsen, Alligatoren ergeben, dass das Leben ohne Brust- und Bauchorgane stundenlang forthestehen kann, wenn für eine genügende Durchblutung des Gehirns Sorge getragen ist, ferner dass die Erscheinungen der Zersetzung und Fäulnis tagelang hintangehalten werden können, wenn frisch verstorbene organlose Individuen durch geeignete Vernähung ihrer Halsgefässe an den Kreislauf eines lebenden Individuums angreschlossen werden. Beim Frosch und bei der Eidechse spielt die Feuchtigkeit eine wichtige Rolle in der Erhaltung des Lebens.

Coakley, Byron, 2. A Report of a few transplantation experiments. Ein gravider Uterus wurde mit Inhalt auf eine Extremität verpflanzt und blieb nuebrere Stunden am Leben, ebenso fuhr ein timonatiger Fötus, der mit seinen Nabelgefässen an die mütterliche Clnararterie angeschlossen war, fort zu leben und zu wachsen. Die übrigen Verpflanzungen nicht nur von Magen, Niereu, Drüsen, sondern von ganzen Köpten, Brustkörben und Körperhälften an Hunden klingen im Bericht so abenteuerlich, dass es fast unmöglich ist, den guten Glauben zu wahren. 
Coakley, Byron, 3. A preliminary report of some experimental work in fractures and dislocations, enthält die Versuche des Verf., durch Injektionen von verschieitenen Substanzen, um die Bruchenden bei Frakturen zur schnellen Vereinigung zu bringen.

K ünne (Steglitz).

Jaquet Albert, Zur. Teehnik der Mastisolbehandlung. Deutsche med. Wochensehrift 1913, Nr. 42.

Verf. bespricht ausführlich die Anwendung des Mastisol, das er sebr warm empfieblt und als ein Idealmittel bexeichuet, dessen Gebrauch einen wirkliehen Fortschritt in der Chirurgie darstellt. Fr stimmt Kausch zu; der Mastisol als ein Universalmittel für die Chirurgie bezeichnet, das Antiseptikum, Heftpflaster und Binden ersetzt.

J. Riedinger.

Iselin, Hans (Basel), Entgiftang des tuberkulösen Herdes dureh Röntgenbestrahlung. Deutsehe med. Wochensehrift, 1913, Nr. $7-8$.

Verf. fasst seine Erfabrungen folgendermassen zusammen:

${ }_{3}$ Regelmässige Gewichtsbestimmungen haben ergeben, 1. dass der Körper in der Röntgenreaktionszeit sein Gewicht in 70-80\%0 der Beobachtungen um etwa $1 \mathrm{~kg}$ nach der Bestrahlung des tuberkulösen Herdes vermehrt, 2. dass sich eine Körpergewichtszunahme fast mit jerler Sitzang wiederholt und dass mit dieser Gewichtszunahme eine Umfangsverminderung in der Gegend des Krankheitsberdes einhergeht.

Der Gewichtsverlust des tuberkulös Erkrankten wird durch Giftbildung im tuherkulösen Herd verursacht.

Die Körpergewichtszunahme, die der Röntgenbelichtung folgt, und die $\mathrm{Ab}$ sehwellung des kranken Gliedes sind als Folgen einer teilweisen Entgiftung des tuberkulösen Herdes durch Bestrahlung autzufassen. Die Gerrichtszunahme kommt vermulich durch vermehrte. Wasseraufnahme und Abschwellung des kranken Gliedes in der Anfangszeit wenigstens duroh Verbeseerung der Zirkulation zustande.

Der weitere Anstieg der Gewichtskurve und die Abnahme der entzündlichen tuberkulösen Schwellung sind ein deutlicher Ausdruck der Heilkraft der fortgesetzten Röntgenhestrahlung.

Der Röntgenbehandlung der chirurgischen Tuberkulose stehen zwei Wege offen: Bei leichten Formen wird sie versuchen, durch wiederholte schwache Belichtung den Ferd zu entgitten und der Resorption zugänglich zu machen.

Bei schwereren Erkrankungen wird, wenn das physiologische Verfabren nicht genügt, eine stärkere Bestrahlung einsetzen müssen; lies bezweckt nicht nur, das tuberkulose Gewebe zum Zerfall zu bringev, sondern sie ist bestrebt, mit energischer Tiefenbestrahlung durch Gefässcebädignng im tuberkulösen Herd and seiner Umgrebung, die Schrumpfung des Granulationsgewebes und Narbenbildung zur Folge hat, die Abkapselung und Aussobaltung des tuberkulösen Herdes aus dem Körper zu fördern.

Bei beiden Arten der Bestrahlung zielt unser Bestreben darauf hin, natürliche Heilungsvorgänge zu unterstützen und zu beschleunigen."

\section{J. Riedinger.}

Max Böm, Die angeborenen Entwickelungsfehlerdes Rumpfskeletts. Berliner klin. Wochenschrift 1913. Nr. 42.

Verf. teilt die angeborenen Deformitäten des Rumpfskelettes ein in 5 Gruppen, nämlich 1. in Formbindungen durch intrauterinen Druck, 2. in Missbildungen durch Defekte und Fusionen, 3. in Varietäten, 4. in Störungen der bilateralen Symmetrie und $\tilde{j}$. in fehlerbafte angeborene Wachstumsrichtung. Schulskoliosen gibt es nicht. Dagegen ist, wie Verf. schon wiederholt zu betonen Gelegenbeit batte, das grosse Heer der nicht entaündichen und nicht paralytischen fixierten 
Rumpfileformitäten, soweit nicht Rachitis vorliegt, in der Hanptsache durch an. geborenen Eutwicklungsfebler des Rumpfskeletts bedingt. J. Riedinger.

Gottstein, J. F. (Reichenberg), Die Skolinse im Leben und in der ärztlichen Praxis. Prager men. Wochenschrift 1913, Nr. 11.

Verf. beklagt es, dass die Mediziner während ihrer Studienzeit gowenig über Skoliose hören, obwohl von vierzehnjährigen Kindern der vierte Teil mit dieser Erkrankung behattet ist. Er geht näber auf die von Irzten bäufig begangenen proguostischen und therapeutiscben Irrtümer hin. An Selbstheilung dürfen wir nie denken. Deshalh muss die Therapie frübzeitigr eingeleitet werdeu. Die Wege und Mittel sind aber verschieden. Verf. verlangt, dass die Orthopüdie selbststïndiges Lehr- und Prüfungsfach an den Universitäten werde. Ausserdem wirl die Aufstellung von Fürsorgeärzten, Praktikern, die nlle gesundheithichen Verhäl. nisse der Kinder uud ihrer häulichen Cmgohung au überwachen, empfolsten. Das wäre eine cier Aufgabe, der sich die hrüppeltürsorge zu widmen hälte.

$$
\text { J. Riedinger. }
$$

Huhtek, Johunn, Ischias kyphotica. Deutsche med. Wochenschrift 1913, Nr. +1 .

Ein 38 jähriger Mann, der frei von jeder nerrösen Belastung war, erkrankte im Anschlusse an eine Überanstrengung an einer typischen rechtseitigen Ischias, die nil einer Kyphose von $120^{\circ}$ einherging. Bei Nacht musste er, um die Schmerzen zu liulern, die Position à la vache einnebmen. Durch die Beugung des Körpers nach vorn wurde der Schmerz beseitigt oder wenigstens gelindert, woraus geschlossen werden muss, dass in den Rückenmuskeln, wahrscheinlich in M. sacrolumbalis, ein stärkerer sensitiver Ast des $\mathrm{N}$. ischiadicus verläuft; dieser Ast nahm an dem pathologischen Prozess teil und litt unter dem Druck der kontrahierten Muskeimasse.

J. Riedinger.

Franz Rost, Experimentale und klinische Untersucbungen über chronische, granulierende Entzündungen des Knocheumarkes. Deutsche Zeitschrift für Chirurgie, Bd. 125.

Ausgehend von dem Studium der noeb wenig aufgeklärten, nicht tuberkulösen, chroniachen Osteomyelitis, und in der Erkenntnis, dass gerade das Knocbenmark sehr geeignet ist, um zu prïfen, ob ein Stoff zur Bindegewebsbildung anregt oder nichr, unternahm Verf. zablreiche Experiniente an Kaninchen und Hunden, un physikalische, chemische und bakterielle Reize auf das Kuochenmark einwirken zu lassen. Zu diesen Zwecke wurden zuücht mechanisch reizende Scotfe in das Knochenmark eingespritat. Die Veränderungen und die bistologisehen Befunde werden ausführlich besprochen. Besonders benerkenswert ist, dass Wismut zur Bindegewebsbildung vicht auregt und deshalb ganz unschuldig ist an der Heilwirkung der Beck schen Paste. Als wirksame Substanz in der Paste kann nur die amerikanische Vaseline angesehen werden.

Unter den rein chemisch wirkenden, d. h. völlig löslichen Stoffen zeichnet sich in hervorragender Weise Rohparaffinol durch die Fähigkeit aus, Bindewucherung hervorzurufen. Verf. hat dieses Mittel auch zu therapeutischen $Z$ wecken bei Osteomyelitis in der chirurgischen Klinik zu Heidelberg verwendet und ist mit den Erfolgen sebr zufrietien. Grosse Defekte nach Sequesteotomie füllen sich schnell mit Granulationen. Bei Empyemeu liess schnell die Eiterung nach und die Höhlen verkleinerten sich. Die Einspritzung abgetöteter Mikrokokken bewirkte tumorartige Granulatiousbildungen, wie sie ähnlich auch beim Menschen zu finden sind (Osteomyelitis und Ostitis fibrosa). Zur Feststellung des Charakters dieser Bildungen weist Verf. auf die Wichtigkeit der serologischen Untersuchung hin. Zum Belege hierfür teilt Verf. eine Reihe klinischer Beobachtungen am Scbiusse seiner interessanten und wichtigen Arbeit mit. In bezug hierauf wäre nocb zu er- 
wähnen, dass aucb ein Fall von juveniler Arthritis der Hüte als Staphylomykose angesprochen werden konnte.

J. Riedinger.

Erich Klose (Breslau), Zur Kenntnis der Osteops thy rosis idiopathica. Monatsschr. für Kinderheilkunde, Bd. XII, 1913.

Verf. hat im Krüppelbeim Zwiukau-Marienthal (Dr. Gaugele) 4 Fälle von Osteopsothyrosis idiopathica beobachtet, über die er ausführlich berichtet. Die wesentlicben Züge des charakteristischen klinischen Bildes werden hervorgeboben. Besonders sorgfilltig werden die Röntgenbefunde beschrieben. Ausgehend von den anatomischen Untersuchungen Loosers tritt Verf. auch der Frage näher, ob sich die verschiedenen radiologischen Befunde mit den anatomischen Untersuchungen in Ubereinstimmung bringen lassen. Der 4. Fall liess diese Übereinstinmung nicht erkennen. Es fehlte hier die für die Osteopsathyrosis idiopathica pathognomonische Verschmächtigung der Driaphysen. Ausserdem ergaben die strukturverhältnisse ein wesentich anderes Bill. Am bemerkenswertesten sind die völlige Strukturlosigkeit mit wechselnder Schattentiefe, eine hochgradig gesteigerte Strahlendurchlässigkeit und der Omstand, lass die Epiphysen geringere Veränderungen zeigen als die Diaphysen. Auch die histologischen Untersuchungen, die Prof. $R$ isel an einem der Tibia durch Osteotomie gewonnenen Knochenstück vornahm und über die er am Schlusse der Arbeit selbst umgehend berichtet, geht hervor, dass dem Fall, der klinisch zum Kraukheitsbild der idiopathischen Osteopsathyrosis zu rechnen ist, ein andersartiger pahologischanatomischer Prozess zugrunde liegt. Die Deutung des Befundes begegnet noch grosen Schwierigkeiten. Im allgemeinen besteht eine Ähnlichkeit mit der Ostitis fibrosa, die sich nach v. Recklinghausen auf dem Boden der chronischen Osteomalazie an einzelnen oder nehreren Abschnitten des Skeletts unter der Einwirkung chronischer Reize entwickelt. Die relativ breiten osteoiden Säume an den Knochenbälkchen deuter $\mathrm{R}$ is el nicht als Halisterese kalkhaltigen Knochens, sondern als Apposition von kalklosem Gewebe durch die Osteoblasten. Demnach handelt es sich um eine Anonalie des Verkalkungsprozesses, mangehatite Kalkablagerung in der Knochensubstanz, wie sie sowohl der Rachitis als der Osteomalazie zukomme. Näheren Autschluss wird nur das Studium der endocbondralen Ossifikation bringen.

J. Riedinger.

Uber die Frage der Gewhihung an Unfallfolgen sprach sich der Prisident des bayerischen Landesversieherungsamtes in der Kammer der Abgeorlneten des hayerischen Landtages am 1U. Mär 1914 folgendermassen aus:

Die. Gewöhung ist ein wahres Kreuz her Rechtsprechung geworden. Wir stehen jetzt auch auf den Standpunkt, dass die Gewöhnung doch nicht in dem horrenden Nasse ausfallen soll wie bisher. Bekanntich hat es damit angefangen, dass die Eisen- und Stahberuf stellung von Fällen brachte, in denen trotz Verlustes einer Hand oder eines anderen Gliedes der Arbeiter später mit gleichem Lohne beschäftigt wurde. Die Arzte sind ebenfalls nachgeschritten und so hat sich eine gewisse Latitüde in der Beurteilung der Gewöhnung herausgebillet. Wir sind $z$. B. so weit gegangen, dass wir sagten: Bei Frauen, bei denen der Zeigefinger eine ganz enorme Bedeatung für die Hruswirtschaft besitzt, kann man bei dessen Verlust eine Gewöhnung nicht mehr annebmen. Die $10 \%$ müssen da bezahit werden, nach wie vor. So baben wir schon verschiedene solche Fälle entschieden. Was daun die Gewöbnung z. B. bei gauz alten Leuten betrifft, so ist es einfach so: Wenn der Mann zur Arbeit unfähig ist, dann kann er eben auch trotz Gewöhnung nicbt. arbeiten. 